\title{
Thermodynamic information geometry and complexity growth of a warped AdS black hole and the warped $\mathrm{AdS}_{3} / \mathrm{CFT}_{2}$ correspondence
}

\author{
H. Dimov, ${ }^{1,2, *}$ R. C. Rashkov, ${ }^{1,3, \dagger}$ and T. Vetsov ${ }^{1,2, \$}$ \\ ${ }^{1}$ Department of Physics, Sofia University, 5 J. Bourchier Blvd., 1164 Sofia, Bulgaria \\ ${ }^{2}$ The Bogoliubov Laboratory of Theoretical Physics, JINR, 141980 Dubna, Moscow region, Russia \\ ${ }^{3}$ Institute for Theoretical Physics, Vienna University of Technology, \\ Wiedner Hauptstr. 8-10, 1040 Vienna, Austria
}

(Received 5 March 2019; published 13 June 2019)

\begin{abstract}
We study the thermodynamic properties of a warped $\mathrm{AdS}_{3}$ black hole within the framework of thermodynamic information geometry. Our analysis focuses on finding the set of proper thermodynamic Riemannian metrics on the space of equilibrium states, together with the conditions for local and global thermodynamic stability. We use our findings to constrain the values of left and right central charges from the dual conformal field theory and the parameters of the bulk gravitational theory.
\end{abstract}

DOI: $10.1103 /$ PhysRevD.99.126007

\section{INTRODUCTION}

In the past few decades the AdS/CFT correspondence revealed important perturbative and nonperturbative phenomena in various classical and quantum models. One of its important features is that it relates a classical gravitational theory in the bulk of spacetime to a quantum theory without gravity on a lower-dimensional boundary, and vice versa. On the other hand, the correspondence is also a duality between weak/strong coupling regimes of both theories.

A specific example of such a duality is given by the correspondence between a warped three-dimensional (3D) anti-de Sitter black hole and a two-dimensional conformal field theory (CFT). In this case, the warped AdS black hole is a stable vacuum solution of the $3 \mathrm{D}$ topological massive gravity (TMG), described by the action [1]

$I_{\mathrm{TMG}}=\frac{1}{16 \pi G} \int_{\mathcal{M}} d^{3} x \sqrt{-g}\left(R+\frac{2}{L^{2}}\right)+\frac{1}{\mu} I_{\mathrm{CS}}+\int_{\partial \mathcal{M}} B$.

Here, $I_{\mathrm{CS}}$ is the gravitational Chern-Simons action,

$I_{\mathrm{CS}}=\frac{1}{32 \pi G} \int_{\mathcal{M}} d^{3} x \sqrt{-g} \varepsilon^{\lambda \mu \nu} \Gamma_{\lambda \sigma}^{r}\left(\partial_{\mu} \Gamma_{r \nu}^{\sigma}+\frac{2}{3} \Gamma_{\mu \tau}^{\sigma} \Gamma_{\nu r}^{\tau}\right)$,

\footnotetext{
*h_dimov@phys.uni-sofia.bg †rash@phys.uni-sofia.bg

*vetsov@phys.uni-sofia.bg
}

Published by the American Physical Society under the terms of the Creative Commons Attribution 4.0 International license. Further distribution of this work must maintain attribution to the author(s) and the published article's title, journal citation, and DOI. Funded by SCOAP. and the coupling $\mu$ is the mass of the graviton, $\varepsilon^{\lambda \mu \nu}=\epsilon^{\lambda \mu \nu}$ / $\sqrt{-g}, \epsilon^{012}=+1$. The boundary term,

$$
B=\frac{1}{32 \pi G} \epsilon_{A B C} \omega^{A B} \wedge e^{C},
$$

was introduced in [2] to make the variational principle well defined. For every value of the coupling $\mu$ TMG has a classical $\mathrm{AdS}_{3}$ solution with a radius $L$. The only stable case is defined by the condition $\mu L=1$, which leads to a non-negative energy of the gravitons. In this case, it is also possible to construct a consistent quantum theory of the so-called chiral gravity [3].

However, if we consider nonchiral values of $\mu L$, we can construct other stable TMG vacua, namely warped backgrounds. They are discrete quotients by elements of $S L(2, R) \times U(1)$ of warped $\mathrm{AdS}_{3}$ space. In this particular case, the group elements of the quotient select the left and the right temperatures of the corresponding boundary CFT. With a suitable choice of the central charges the density of states in the boundary CFT exactly matches the BekensteinHawking entropy of the corresponding black hole; thus a duality between both theories can be conjectured. It is also worth mentioning that warped $\mathrm{AdS}_{3}$ solutions arise in a number of contexts besides TMG; see e.g., [4-12].

Recently, the holographic principle was used to describe the complexity of a quantum state via bulk theory computations. In this case, there are two conjectures. The first one is called "complexity equals action" (CA) [13,14], which states that in order to compute the quantum computational complexity of a holographic state, one can calculate the onshell action on the so-called "Wheeler-De Witt" patch:

$$
C_{A}(\Sigma)=\frac{I_{\mathrm{WDW}}}{\pi \hbar}
$$


where $\Sigma$ is the time slice intersection of the asymptotic boundary and the Cauchy surface in the bulk. The second proposal, known as "complexity equals volume" (CV) [15-17], relates the complexity of the boundary states with the volume $V$ of a maximal slice behind the event horizon, i.e.,

$$
C_{V}(\Sigma)=\max _{\Sigma=\partial B}\left[\frac{V(B)}{G_{N} \ell}\right] .
$$

A nice feature of the $\mathrm{CV}$ conjecture is that the maximal volume naturally grows at a rate proportional to the product between the temperature $T$ and the entropy $S$ of the black hole. Moreover, the idea that the growth of complexity can be interpreted as a computation naturally invokes Lloyd's bound [18] on the rate of computation for systems with energy $M$ :

$$
\frac{d C}{d t} \leq \frac{2 M}{\pi \hbar}
$$

Furthermore, several other information-theoretic concepts have been fruitfully applied to the investigation of fundamental properties of various gravitational systems, namely entanglement entropy [19-23], Fisher information metric [22,23], and thermodynamic information geometry (TIG) [24-33]. The latter can be considered as a specific thermodynamic limit of the quantum Fisher metric. The common belief is that the classical spacetime geometry has the capacity to encode important properties of the dual quantum system. Due to the lack of a consistent theory of quantum gravity a case by case study is required. Here, we will focus on the thermal properties of the warped $\mathrm{AdS}_{3}$ black hole via the methods of TIG.

Thermodynamic information geometry was first introduced by Weinhold [34] and later by Ruppeiner [35]. Weinhold showed that the empirical laws of equilibrium thermodynamics can be brought into correspondence with the mathematical axioms of an abstract metric space. In his approach, Weinhold used the Hessian of the internal energy with respect to the extensive parameters of the system in order to introduce a Riemannian metric on the space of macro states,

$$
g_{a b}^{(W)}=\partial_{a} \partial_{b} U\left(E^{c}\right) .
$$

Here $E^{c}$ are the other extensive parameters of the system besides $U$. On the other hand, Ruppeiner developed his geometric approach within fluctuation theory, where one implements the entropy $S\left(E^{c}\right)$ as a thermodynamic potential in order to define a Hessian metric structure on the state space statistical manifold:

$$
g_{a b}^{(R)}=-\partial_{a} \partial_{b} S\left(E^{c}\right) .
$$

Here $E^{c}$ are the other extensive parameters of the system besides $S$.

The importance of using Hessian metrics on the equilibrium manifold is best understood when one considers small fluctuations of the thermodynamic potential. The potential is extremal at each equilibrium point, while the second moment of the fluctuation turns out to be directly related to the components of the corresponding Hessian metric. As it turned out, both metric approaches are conformally related to each other with the temperature being the conformal factor,

$$
d s_{(R)}^{2}=\frac{1}{T} d s_{(W)}^{2} .
$$

Although Weinhold and Ruppeiner metrics have been successfully applied to describe the phase structure of condensed matter systems, when utilized for black holes they do not often agree with each other. One of the reasons is due to the fact that Hessian metrics are not Legendre invariant, and thus they do not preserve the geometric structure when a different thermodynamic potential is used for the description of the equilibrium states. In order to make things Legendre invariant, Quevedo considered the $(2 n+1)$-dimensional thermodynamic phase space, spanned by the thermodynamic potential $\Phi$, the set of extensive variables $E^{a}$, and the set of intensive variables $I^{a}$, $a=1, \ldots, n$. In Ref. [36] it was found that the general metric on the equilibrium state space can be written in the form

$$
g^{I, I I}=\Omega_{\Phi} \Phi\left(E^{c}\right) \chi_{a}^{b} \frac{\partial^{2} \Phi}{\partial E^{b} \partial E^{c}} d E^{a} d E^{c},
$$

where $\chi_{a}{ }^{b}=\chi_{a f} \delta^{f b}$ is a constant diagonal matrix and $\Omega_{\Phi} \in \mathbb{R}$ is the degree of generalized homogeneity, $\Phi\left(\lambda^{\beta_{1}} E^{1}, \ldots, \lambda^{\beta_{N}} E^{N}\right)=\lambda^{\Omega_{\Phi}} \Phi\left(E^{1}, \ldots, E^{N}\right), \beta_{a} \in \mathbb{R}$. In this case, the Euler identity for homogeneous functions is given by

$$
\beta_{a b} E^{a} \frac{\partial \Phi}{\partial E^{b}}=\Omega_{\Phi} \Phi
$$

where $\beta_{a b}=\operatorname{diag}\left(\beta_{1}, \beta_{2}, \ldots, \beta_{N}\right)$. From the first law $d \Phi=I_{a} d E^{a}$, one notes that $I_{a}=\partial \Phi / \partial E^{a}$. When $\beta_{a b}=\delta_{a b}$, one returns to the standard Euler's identity. If we choose $\beta_{a b}=\delta_{a b}$, for complicated systems this may lead to some nontrivial conformal factor that is no longer proportional to the potential $\Phi$. On the other hand, if we set $\chi_{a b}=\delta_{a b}$, the resulting metric $g^{I}$ can be used to investigate systems with at least one first-order phase transition. Alternatively, the choice $\chi_{a b}=\eta_{a b}=\operatorname{diag}(-1,1, \ldots, 1)$, leads to a different metric $g^{I I}$, which applies to systems with second-order phase transitions.

Consequently, one can show that the corresponding scalar thermodynamic curvature encodes information about 
the phase structure of the system. As suggested by Ruppeiner in Ref. [35], the Ricci information curvature $R_{I}$ is related to the correlation volume of the system. This association follows from the idea that it will be less probable to fluctuate from one equilibrium thermodynamic state to the other, if the distance between the points on the statistical manifold, which correspond to these states, is larger. Moreover, the sign of the scalar curvature can be linked to the nature of the interparticle interactions [37]. For example, if $R_{I}=0$, the interactions are absent and the system is free. For positive curvature the interactions are repulsive, while for negative curvature the interactions are attractive.

Finally, the scalar curvature on the equilibrium manifold can also be used to measure the stability of the physical system under thermodynamic perturbations. In particular, the information curvature approaches infinity in the vicinity of critical points, where phase transitions occur [38]. Moreover, the curvature of the information metric tends to diverge not only at the critical points of phase transitions but also on entire regions of points on the statistical manifold, called spinodal curves. The latter can be used to discern physical from nonphysical situations.

In this paper we investigate the properties of a warped $\mathrm{AdS}_{3}$ black hole solution of TMG within the framework of thermodynamic information geometry. The text is structured as follows: In Sec. II we introduce the warped $\mathrm{AdS}_{3}$ black hole and the relevant thermodynamic quantities. In Sec. III we calculate the heat capacity of the warped $\mathrm{AdS}_{3}\left(\mathrm{WAdS}_{3}\right)$ black hole in different parameter spaces and analyze the conditions for local thermodynamic stability of the system. In Sec. IV we calculate the Hessian thermodynamic metrics on the space of equilibrium states and show that they fail as viable Riemannian metrics. Also, in this section we identify the regions of global thermodynamic stability in canonical and grand canonical ensembles. In Sec. V we investigate the properties of two conformally related Legendre invariant metrics, namely Quevedo and Hendi-Panahiyan-EslamMomennia (HPEM) information metrics. We show that they successfully reproduce the Davies phase transition points and thus can be used to describe the space of equilibrium states. In Sec. VI we investigate a third approach for introducing thermodynamic metrics on the equilibrium manifold by defining various conjugate thermodynamic potentials. By construction, the thermodynamic metrics in this approach are designed to match the Davies transition points of the heat capacity, without any additional singularities. We confirm that this is also valid for the $\mathrm{WAdS}_{3}$ case. In Sec. VII we calculate the complexity growth of the warped solution and show that it is compatible with the conditions for thermodynamic stability. Furthermore, we find that Lloyd's bound on the complexity growth leads to a lower bound on the mass of the black hole. Finally, in Sec. VIII we make brief comments on our results.

\section{WARPED AdS ${ }_{3}$ BLACK HOLE AND ITS THERMODYNAMICS}

The warped $\mathrm{AdS}_{3}$ solution of (1.1) is given by the following metric [1]:

$$
\begin{aligned}
\frac{d s^{2}}{L^{2}}= & d t^{2}+\frac{d r^{2}}{\left(\nu^{2}+3\right)\left(r-r_{+}\right)\left(r-r_{-}\right)} \\
& +\left(2 \nu r-\sqrt{r_{+} r_{-}\left(\nu^{2}+3\right)}\right) d t d \theta \\
& +\frac{r}{4}\left(3\left(\nu^{2}-1\right) r+\left(\nu^{2}+3\right)\left(r_{+}+r_{-}\right)\right. \\
& \left.-4 \nu \sqrt{r_{+} r_{-}\left(\nu^{2}+3\right)}\right) d \theta^{2},
\end{aligned}
$$

where $r \in[0, \infty], t \in[-\infty, \infty]$, and $\theta \sim \theta+2 \pi$. The horizons are located at $r_{+}$and $r_{-}$, where $1 / g_{r r}$ as well as the determinant of the $(t, \theta)$ metric vanishes. Here, we also introduced the parameter $\nu=\mu L / 3$. Notice that (2.1) reduces to the Banados-Teitelboim-Zanelli (BTZ) black hole in a rotating frame, when $\nu^{2}=1$. For $\nu^{2}>1$, we have physical black holes, as long as $r_{+}$and $r_{-}$stay positive. For $\nu^{2}<1$, we always encounter closed timelike curves and such geometries will not be considered. The Ricci curvature and the Kretschmann scalar invariant of the metric are

$$
R=-\frac{6}{L^{2}}, \quad K=6 \frac{3-2 \nu^{2}+\nu^{4}}{L^{4}}
$$

Nonphysical situations occur at $L=0$ and $\nu \rightarrow \pm \infty$, and thus it is natural to consider $L>0$ and finite $\nu^{2}>1$ for the nonchiral case.

The entropy $S$ of the warped AdS black hole and the Abbott-Deser-Tekin (ADT) conserved charges $M$ and $J$ are given by

$S=\frac{\pi L}{24 \nu G}\left(\left(9 \nu^{2}+3\right) r_{+}-\left(\nu^{2}+3\right) r_{-}-4 \nu \sqrt{\left(\nu^{2}+3\right) r_{+} r_{-}}\right)$,

$$
M=\frac{\left(\nu^{2}+3\right)}{24 G}\left(r_{+}+r_{-}-\frac{1}{\nu} \sqrt{r_{+} r_{-}\left(\nu^{2}+3\right)}\right),
$$

and

$$
\begin{aligned}
J= & \frac{\nu L\left(\nu^{2}+3\right)}{96 G}\left[\left(r_{+}+r_{-}-\frac{1}{\nu} \sqrt{r_{+} r_{-}\left(\nu^{2}+3\right)}\right)^{2}\right. \\
& \left.-\frac{\left(5 \nu^{2}+3\right)}{4 \nu^{2}}\left(r_{+}-r_{-}\right)^{2}\right] .
\end{aligned}
$$

One can also compute the Hawking temperature and the angular velocity: 


$$
\begin{aligned}
& T=\frac{\left(\nu^{2}+3\right)\left(r_{+}-r_{-}\right)}{4 \pi L\left(2 \nu r_{+}-\sqrt{\left(\nu^{2}+3\right) r_{+} r_{-}}\right)} \\
& \Omega=\frac{2}{L\left(2 \nu r_{+}-\sqrt{\left.\left(\nu^{2}+3\right) r_{+} r_{-}\right)}\right.} .
\end{aligned}
$$

In this case, the first law of thermodynamics holds,

$$
d M=T d S+\Omega d J
$$

Here, it is relevant to comment on whether the thermodynamic quantities can become zero. Considering $r_{+} \geq r_{-}$, one can reach $S=0$ only if $-1 \leq \nu \leq 1$, which is excluded. The same condition holds for $M=0$. Thus, we consider only positive $S>0, M>0$. From Eq. (2.6) one notes that the angular velocity $\Omega$ cannot be zero, while the temperature $T$ is zero for coincident horizons, $r_{+}=r_{-}$. On the other hand, the laws of thermodynamics forbid us from ever reaching absolute zero; thus $T>0$. The only quantity that can become zero within $\nu^{2}>1$ is $J$. Without loss of generality, we will consider only non-negative values of the angular charge, $J \geq 0$.

Instead of $r_{+}$and $r_{-}$, we can work with the left and right temperatures, namely

$$
\begin{aligned}
& T_{R}=\frac{\left(\nu^{2}+3\right)\left(r_{+}-r_{-}\right)}{8 \pi L}, \\
& T_{L}=\frac{\left(\nu^{2}+3\right)}{8 \pi L}\left(r_{+}+r_{-}-\frac{\sqrt{\left(\nu^{2}+3\right) r_{+} r_{-}}}{\nu}\right),
\end{aligned}
$$

and the left and right central charges,

$$
\begin{aligned}
c_{R} & =\frac{\left(5 \nu^{2}+3\right) L}{G \nu\left(\nu^{2}+3\right)}, \quad c_{L}=\frac{4 \nu L}{G\left(\nu^{2}+3\right)}, \\
c_{L}-c_{R} & =-\frac{L}{G \nu},
\end{aligned}
$$

of the dual $\mathrm{CFT}_{2}$ theory. Although we are going to consider only positive central charges throughout the paper, which lead to unitary CFTs, one should keep in mind that negative charges can play a vital role in anomaly cancellations, when considering the total central charge. From Eq. (2.9), under the requirement of positive central charges and $\nu^{2}>1$, one can restrict only to $\nu>1$. Therefore, it immediately follows that $c_{L}<L$ and $c_{R}<2 L$. For large $\nu \rightarrow \infty$ one has vanishing central charges, which is physically excluded due to the divergence of the Kretschmann invariant (2.2). Furthermore, the third expression in Eq. (2.9) clearly forbids the case $c_{L}=c_{R}$, while its negative sign suggests that $c_{R}>c_{L}$. The comments above show that

$$
0<c_{L}<L, \quad L<c_{R}<2 L \text {. }
$$

On the other hand, from Eq. (2.9), one finds the ratio of the central charges

$$
\frac{c_{L}}{c_{R}}=\frac{4 \nu^{2}}{3+5 \nu^{2}} \text {. }
$$

It depends only on $\nu$ and certain limiting cases are valid. For $\nu \rightarrow \infty$, the ratio reaches a maximum value of 4/5. One has to exclude this value due to Eq. (2.2). When $\nu=1$, the ratio is $1 / 2$, which is also excluded from our considerations. Therefore,

$$
\frac{1}{2}<\frac{c_{L}}{c_{R}}<\frac{4}{5}
$$

In terms of the dual CFT temperatures and charges the entropy takes the Cardy form

$$
S=\frac{\pi^{2} L}{3}\left(c_{L} T_{L}+c_{R} T_{R}\right)
$$

One can also define the following left and right moving energies:

$$
E_{L}=\frac{\pi^{2} L}{6} c_{L} T_{L}^{2}, \quad E_{R}=\frac{\pi^{2} L}{6} c_{R} T_{R}^{2},
$$

which allow us to write the ADT conserved mass $M$ and angular momentum $J$ as

$$
M=\frac{1}{G} \sqrt{\frac{2 L E_{L}}{3 c_{L}}}, \quad J=L\left(E_{L}-E_{R}\right) .
$$

Consequently, the Hawking temperature $T$, defined as the surface gravity of the horizon divided by $2 \pi$, yields

$$
\frac{1}{T}=\frac{4 \pi \nu L}{\nu^{2}+3} \frac{T_{L}+T_{R}}{T_{R}} .
$$

In order to study the thermodynamic properties of the WAdS black hole, we will express the entropy $S$ in terms of the other extensive parameters $M$ and $J$, and also the ADT mass $M$ in terms of $S$ and $J$. From Eqs. (2.13)-(2.16) one finds the explicit expressions $(G=1)$

$$
S(M, J)=\pi\left(M c_{L}+\sqrt{c_{R}\left(c_{L} M^{2}-\frac{2 J}{3}\right)}\right)
$$

and

$M(S, J)=\frac{\sqrt{3 c_{L} c_{R}\left(2 \pi^{2} J\left(c_{R}-c_{L}\right)+3 S^{2}\right)}-3 c_{L} S}{3 \pi c_{L}\left(c_{R}-c_{L}\right)}$.

It will be convenient for our considerations to also express $S$ and $M$ in terms of other parameters, namely in $(J, T)$ space:

$$
S(J, T)=\frac{\pi \sqrt{2 c_{L} J}\left(1+\left(c_{R}-c_{L}\right) \pi T\right)}{\sqrt{3} \sqrt{1-c_{L} \pi T\left(2+\left(c_{R}-c_{L}\right) \pi T\right)}},
$$


$M(J, T)=\frac{\sqrt{2 J}\left(1-c_{L} \pi T\right)}{\sqrt{3 c_{L}\left(1-c_{L} \pi T\left(2+\left(c_{R}-c_{L}\right) \pi T\right)\right)}}$.

We can also take advantage of the first law (2.7) to find $T$ and $\Omega$ in $(S, J)$ space,

$$
\begin{aligned}
T(S, J) & =\frac{\partial M}{\partial S} \\
& =\frac{1}{\pi\left(c_{L}-c_{R}\right)}\left(1-\frac{\sqrt{3 c_{R}} S}{\sqrt{c_{L}\left(2 J \pi^{2}\left(c_{R}-c_{L}\right)+3 S^{2}\right)}}\right),
\end{aligned}
$$

$\Omega(S, J)=\frac{\partial M}{\partial J}=\frac{\pi c_{R}}{\sqrt{3 c_{L} c_{R}\left(2 J \pi^{2}\left(c_{R}-c_{L}\right)+3 S^{2}\right)}}$,

or, equivalently, in $(M, J)$ space

$$
\begin{gathered}
T(M, J)=\frac{1}{c_{L} \pi}\left(1+\frac{\sqrt{3 c_{R}} M}{\sqrt{3 c_{L} M^{2}-2 J}}\right)^{-1}, \\
\Omega(M, J)=\frac{c_{R}}{c_{L}\left(3 c_{R} M+\sqrt{3 c_{R}\left(3 c_{L} M^{2}-2 J\right)}\right)} .
\end{gathered}
$$

Naturally one requires $T>0$, which constrains the values of the left central charge:

$$
c_{L}>\frac{2 J}{3 M^{2}}=\frac{2 a}{3},
$$

where $a=J / M^{2}$ is the spin parameter. Relation (2.25) shows that for a slowly rotating system, $a<1$, the left central charge $c_{L}$ does not exceed $2 / 3$. For ultraspinning black holes, $a \geq 1$, the charge $c_{L}$ is bounded from above only by $L$; thus we can constrain the rotation of the $\mathrm{WAdS}_{3}$ solution such as

$$
a<\frac{3 L}{2} \text {. }
$$

In the sections below, we investigate the local and global thermodynamic properties of the $\mathrm{WAdS}_{3}$ black hole solution.

\section{HEAT CAPACITY AND LOCAL THERMODYNAMIC STABILITY}

One can distinguish two types of phase transitions with respect to the heat capacity $C$. The first type occurs when $C$ changes sign, i.e., $\partial_{S} M=0$, while the second type is identified by the singular points of the heat capacity itself, i.e., $\partial_{S S}^{2} M=0$. The heat capacity of the $\mathrm{WAdS}_{3}$ black hole in $(S, J)$ space is given by

$$
C(S, J)=\frac{\partial_{S} M}{\partial_{S S}^{2} M}=\frac{\left(2 \pi^{2} J\left(c_{R}-c_{L}\right)+3 S^{2}\right)\left(\sqrt{3} c_{R} S-\sqrt{c_{L} c_{R}\left(2\left(c_{R}-c_{L}\right) J \pi^{2}+3 S^{2}\right)}\right)}{2 \sqrt{3} \pi^{2} J c_{R}\left(c_{R}-c_{L}\right)},
$$

where $J=0$ is a true singularity, while $c_{L}=c_{R}$ is excluded due to Eq. (2.9). The heat capacity changes signs on the following spinodal curve:

$$
2 \pi^{2} J c_{L}-3 S^{2}=0
$$

while $C=0$ occurs on (3.2) or the curve

$$
2 \pi^{2} J\left(c_{R}-c_{L}\right)+3 S^{2}=0 .
$$

Considering the assumptions from the previous section, these curves are not reachable by any macro state of the black hole. The condition for local thermodynamic stability, $C>0$, leads to an upper thermodynamic bound on the left central charge

$$
c_{L}<\frac{3 S^{2}}{2 \pi^{2} J}
$$

Combining Eqs. (3.4) and (2.25), one finds the following relation between the gravitational parameters:

$$
\frac{J^{2}}{M^{2} S^{2}}<\frac{9}{4 \pi^{2}}
$$

The local TD stability in $(M, J)$ coordinates requires the explicit form of the heat capacity,

$C(M, J)$

$$
=\frac{\pi \mathrm{c}_{L} H\left(\mathrm{c}_{R} \sqrt{\mathrm{c}_{R}\left(3 \mathrm{c}_{L} M^{2}-2 J\right)}-\mathrm{c}_{L} \sqrt{\mathrm{c}_{R} H}+\sqrt{3} \mathrm{c}_{L} \mathrm{c}_{R} M\right)}{2 \sqrt{3} \mathrm{c}_{R} J\left(\mathrm{c}_{R}-\mathrm{c}_{L}\right)},
$$

where

$$
H=M\left(2 \sqrt{3 \mathrm{c}_{R}\left(3 \mathrm{c}_{L} M^{2}-2 J\right)}+3 M\left(\mathrm{c}_{L}+\mathrm{c}_{R}\right)\right)-2 J .
$$

As expected, $J=0$ is a true singularity, while $C$ changes sign on 


$$
3 M^{2} c_{L}-2 J=0 .
$$

Imposing $C>0$, one finds agreement with (2.25).

In $(J, T)$ space the heat capacity takes the form

$C(J, T)=T \frac{\partial S(J, T)}{\partial T}=\frac{c_{R} \pi^{2} T \sqrt{2 c_{L} J}}{\sqrt{3}\left(1-c_{L} \pi T\left(2+\left(c_{R}-c_{L}\right) \pi T\right)\right)^{3 / 2}}$.

When $J=0$, the specific heat $C$ changes sign, while it diverges at the following critical temperature:

$$
T_{c}=\frac{1}{\pi\left(c_{L}+\sqrt{c_{L} c_{R}}\right)} .
$$

The local thermodynamic (TD) stability, $C>0$, requires

$$
T<T_{c}, \quad J>0 .
$$

Finally, the heat capacity in $(S, \Omega)$ space is written by

$$
C(S, \Omega)=\frac{\mathrm{c}_{R} \pi^{2}(\pi-3 S \Omega)}{3 \Omega\left(9 \mathrm{c}_{L} S^{2} \Omega^{2}-\pi^{2} \mathrm{c}_{R}\right)} .
$$

It changes sign at

$$
\Omega S=\frac{\pi}{3},
$$

while its divergences are located on

$$
\Omega^{2} S^{2}=\frac{\mathrm{c}_{R} \pi^{2}}{9 \mathrm{c}_{L}} .
$$

The singularity at $\Omega=0$ is excluded due to Eq. (2.6). The local TD stability in $(S, \Omega)$ space defines the region

$$
\frac{\pi}{3}<\Omega S<\frac{\pi}{3} \sqrt{\frac{c_{R}}{c_{L}}} .
$$

Further constraints on the CFT central charges or the gravitational parameters can be found by considering the methods of thermodynamic information geometry, as shown in the following sections. Also, more on the thermodynamic stability of the $\mathrm{WAdS}_{3}$, in the space of the original gravitational parameters, can be found in [39].

\section{HESSIAN THERMODYNAMIC INFORMATION METRICS}

\section{A. Ruppeiner metric}

We begin by calculating the Ruppeiner thermodynamic metric given by the Hessian of the entropy

$$
g_{a b}^{(R)}=-\partial_{a} \partial_{b} S(M, J), \quad a, b=(M, J) .
$$

The explicit form of the metric is written by

$$
\begin{aligned}
\hat{g}^{(R)} & =\left(\begin{array}{cc}
g_{M M}^{(R)} & g_{M J}^{(R)} \\
g_{J M}^{(R)} & g_{J J}^{(R)}
\end{array}\right) \\
& =\left(\begin{array}{cc}
\frac{2 \pi J c_{L} \sqrt{3 c_{R}\left(3 c_{L} M^{2}-2 J\right)}}{\left(3 c_{L} M^{2}-2 J\right)^{2}} & -\frac{\sqrt{3} \pi M_{L} c_{R}^{2}}{\sqrt{\left(c_{R}\left(3 c_{L} M^{2}-2 J\right)\right)^{3}}} \\
-\frac{\sqrt{3} \pi M c_{L} c_{R}^{2}}{\sqrt{\left(c_{R}\left(3 c_{L} M^{2}-2 J\right)\right)^{3}}} & \frac{\pi c_{R}^{2}}{\sqrt{3} \sqrt{\left(c_{R}\left(3 c_{L} M^{2}-2 J\right)\right)^{3}}}
\end{array}\right) .
\end{aligned}
$$

In order to identify any critical points and phase transitions we investigate the singularities of the thermodynamic curvature with respect to the metric (4.2),

$$
R_{I}^{(R)}=-\frac{\sqrt{3}}{\pi \sqrt{c_{R}\left(3 c_{L} M^{2}-2 J\right)}} .
$$

This expression shows that $R_{I}^{(R)}$ is singular at $c_{L}=2 \mathrm{~J} /$ $\left(3 M^{2}\right)$, but regular for $J=0$. Therefore, the Ruppeiner information metric accounts for only half of the necessary critical points of the heat capacity (3.6), and one cannot use it to fully describe the equilibrium space of the $\mathrm{WAdS}_{3}$ black hole solution.

\section{B. Weinhold metric}

On the other hand, one can consider the Weinhold information metric,

$$
g_{a b}^{(W)}=\partial_{i} \partial_{j} M(S, J), \quad i, j=(S, J),
$$

with components

$\hat{g}^{(W)}=\left(\begin{array}{cc}g_{S S}^{(W)} & g_{S J}^{(W)} \\ g_{J S}^{(W)} & g_{J J}^{(W)}\end{array}\right)=\left(\begin{array}{cc}\frac{2 \sqrt{3} J \pi c_{L} c_{R}^{2}}{h^{3 / 2}} & -\frac{\sqrt{3} \pi c_{L} c_{R}^{2} S}{h^{3 / 2}} \\ -\frac{\sqrt{3} \pi c_{L} c_{R}^{2} S}{h^{3 / 2}} & -\frac{\pi^{3} c_{L} c_{R}^{2}\left(c_{R}-c_{L}\right)}{\sqrt{3} h^{3 / 2}}\end{array}\right)$,

where $h=\mathrm{c}_{L} \mathrm{c}_{R}\left(3 S^{2}+2\left(\mathrm{c}_{R}-\mathrm{c}_{L}\right) J \pi^{2}\right)$. In this case, the Weinhold curvature,

$$
R_{I}^{(W)}=\frac{\sqrt{3} \pi \mathrm{c}_{L}\left(\mathrm{c}_{R}-\mathrm{c}_{L}\right)}{\sqrt{\mathrm{c}_{L} \mathrm{c}_{R}\left(2 \pi^{2} J\left(\mathrm{c}_{R}-\mathrm{c}_{L}\right)+3 S^{2}\right)}},
$$

does not reproduce the singularities of the heat capacity (3.1) in $(S, J)$ space. Therefore, it is also not a suitable Riemannian metric on the equilibrium state space of the $\mathrm{WAdS}_{3}$ black hole.

\section{Ensembles and global thermodynamic stability}

By choosing other potentials, one can construct Hessian thermodynamic metrics, which, in general, are not conformally related to Ruppeiner or Weinhold metrics. For example, in the canonical ensemble we can consider the Helmholtz potential, 


$$
F(M, J)=M-T S=\frac{2 J \sqrt{c_{R}}}{c_{L}\left(3 M \sqrt{c_{R}}+\sqrt{3} \sqrt{3 c_{L} M^{2}-2 J}\right)},
$$

which defines the following Hessian thermodynamic metric:

$$
\begin{aligned}
& g_{M M}^{(H)}=\frac{36 J \sqrt{c_{R}}\left(3 c_{L} M^{2}\left(\left(c_{L}+c_{R}\right) f+2 c_{L} M \sqrt{3 c_{R}}\right)+J\left(c_{L}\left(f-3 M \sqrt{3 c_{R}}\right)-2 c_{R} f\right)\right)}{c_{L}\left(\sqrt{3} f+3 M \sqrt{c_{R}}\right)^{3} f^{3}}, \\
& g_{J J}^{(H)}=-\frac{6\left(f \sqrt{c_{R}}\left(J-6 c_{L} M^{2}\right)+3 \sqrt{3} c_{R} M\left(J-2 c_{L} M^{2}\right)\right)}{c_{L} f^{3}\left(\sqrt{3} f+3 M \sqrt{c_{R}}\right)^{3}}, \\
& \operatorname{denom}\left(R_{I}^{(H)}\right) \\
& =-2 f^{2}\left(\left(c_{L}-2 c_{R}\right) f J-3 c_{L} J M \sqrt{3 c_{R}}\right. \\
& \left.+3 c_{L}\left(c_{L}+c_{R}\right) f M^{2}+6 c_{L}^{2} M^{3} \sqrt{3 c_{R}}\right)^{2} \\
& \times\left(\sqrt{c_{R}} f\left(J-3 c_{L} M^{2}\right)+3 \sqrt{3} c_{R} M\left(J-2 c_{L} M^{2}\right)\right. \\
& \left.-3 c_{R}^{3 / 2} f M^{2}\right)^{2}
\end{aligned}
$$$$
g_{M J}^{(H)}=\frac{18 M \sqrt{c_{R}}\left(3 c_{L} J M \sqrt{3 c_{R}}-\left(c_{L}-2 c_{R}\right) f J-3 c_{L}\left(c_{L}+c_{R}\right) f M^{2}-6 c_{L}^{2} M^{3} \sqrt{3 c_{R}}\right)}{c_{L} f^{3}\left(\sqrt{3} f+3 M \sqrt{c_{R}}\right)^{3}},
$$$$
G(J, T)=\frac{\sqrt{J}}{\sqrt{2 c_{L}}} \sqrt{1-c_{L} \pi T\left(2+\left(c_{R}-c_{L}\right) \pi T\right)} .
$$

is zero, when $c_{L}=2 J /\left(3 M^{2}\right)$, but nonzero for $J=0$. The numerator of the TD curvature is also finite, when $J \rightarrow 0$.

The situation does not improve in the grand canonical ensemble as well, where one works with the Gibbs free energy

$G(M, J)=M-T S-\Omega J=\frac{J \sqrt{3 c_{R}}}{c_{L}\left(M \sqrt{3 c_{R}}+\sqrt{3 c_{L} M^{2}-2 J}\right)}$.

The conditions for global TD stability in the canonical and grand canonical ensembles, respectively, are given by [40]

$$
\frac{\partial^{2} F}{\partial T^{2}}<0, \quad \frac{\partial^{2} G}{\partial T^{2}}<0
$$

Here, the concavity of the Helmholtz free energy $F$ and the Gibbs energy $G$, with respect to the temperature $T$, implies that the corresponding specific heats are both positive. Both conditions (4.15) are satisfied for $T<T_{c}$, which is also the requirement (3.11) for local TD stability. Hence, the $\mathrm{WAdS}_{3}$ black hole solution is locally and globally stable, from the thermodynamic standpoint, in the same temperature interval $0<T<T_{c}$, whereas it is unstable for $T>T_{c}$. Near the critical temperature, $T=T_{c}$, the underlying interparticle interactions become strongly correlated and phase transitions occur. In this case, the equilibrium description of the black hole system breaks down.

\section{LEGENDRE INVARIANT INFORMATION METRICS}

\section{A. Quevedo metrics}

The Quevedo information metric on the equilibrium state space of the $\mathrm{WAdS}_{3}$ solution is given by [36]

$d s_{Q}^{2}=W\left(-\partial_{S}^{2} M d S^{2}+\partial_{J}^{2} M d J^{2}\right)=g_{S S}^{(Q)} d S^{2}+g_{J J}^{(Q)} d J^{2}$,

where the conformal function $W$ has one of the following forms:

$$
W= \begin{cases}S \frac{\partial M}{\partial S}+J \frac{\partial M}{\partial J}, & \text { case I, } \\ S \frac{\partial M}{\partial S}, & \text { case II. }\end{cases}
$$


The first expression for $W$ leads to the following components of the information metric:

$$
g_{S S}^{(Q, I)}=\frac{2 J c_{R}^{2} c_{L}\left(\sqrt{3} \mathrm{c}_{R}\left(\pi^{2} J\left(\mathrm{c}_{R}-\mathrm{c}_{L}\right)+3 S^{2}\right)-3 S \sqrt{h}\right)}{\sqrt{3}\left(\mathrm{c}_{L}-\mathrm{c}_{R}\right) h^{2}},
$$

$g_{J J}^{(Q, I)}=\frac{\pi^{2} c_{R}^{2} c_{L}\left(\mathrm{c}_{R}\left(\pi^{2} J\left(\mathrm{c}_{L}-\mathrm{c}_{R}\right)-3 S^{2}\right)+S \sqrt{3 h}\right)}{3 \mathrm{c}_{L} h^{2}}$,

where $h=\mathrm{c}_{L} \mathrm{c}_{R}\left(3 S^{2}+2\left(\mathrm{c}_{R}-\mathrm{c}_{L}\right) J \pi^{2}\right)$. The denominator of the thermodynamic curvature for case I reads

$\operatorname{denom}\left(R_{I}^{(Q, I)}\right)=6 J^{2} \pi^{2}\left(S\left(\sqrt{3 h}-3 \mathrm{c}_{R} S\right)-\pi^{2} \mathrm{c}_{R} J\left(\mathrm{c}_{R}-c_{L}\right)\right)^{4}$.

It is singular at $J=0$. One notes that the curve (3.2) is not reproduced by $\operatorname{denom}\left(R_{I}^{(Q, I)}\right)$. However, we excluded this curve as a true singularity of the heat capacity. Therefore, we can consider this metric as a viable TD metric, if we treat $J=0$ as a first-order phase transition point.

On the other hand, the metric components in case II are given by

$$
\begin{aligned}
\hat{g}_{(Q, I I)} & =\left(\begin{array}{cc}
g_{S S}^{(Q, I I)} & 0 \\
0 & g_{J J}^{(Q, I I)}
\end{array}\right) \\
& =\left(\begin{array}{cc}
\frac{2 c_{L} c_{R}^{2} J S\left(3 \mathrm{c}_{R} S-\sqrt{3 h}\right)}{\left(c_{L}-\mathrm{c}_{R}\right) h^{2}} & 0 \\
0 & \frac{c_{L} c_{R}^{2} \pi^{2} S\left(\sqrt{3 h}-3_{R} S\right)}{3 h^{2}}
\end{array}\right) .
\end{aligned}
$$

The denominator of the second Quevedo curvature,

$$
\begin{aligned}
\operatorname{denom}\left(R_{I}^{(Q, I I)}\right)= & 6 \sqrt{3} \pi^{2} J^{2} S^{3}\left(2 \pi^{2} \mathrm{c}_{L} J\left(\mathrm{c}_{R}-c_{L}\right)\right. \\
& \left.+3 S^{2}\left(\mathrm{c}_{L}+\mathrm{c}_{R}\right)-2 \sqrt{3 h} S\right)^{3},
\end{aligned}
$$

is singular at $J=0$. It is also singular at the spinodal curve (3.2) of the heat capacity, which we discarded. As discussed in Sec. II, the case $S=0$ is excluded, and thus $R_{I}^{(Q, I I)}$ does not show additional singularities. Therefore, the second Quevedo information metric is a viable thermodynamic metric.

We can now consider the values of the $R_{I}^{(Q, I I)}$. In the region of TD stability, $R_{I}^{(Q, I I)}$ is negative, which defines a hyperbolic thermodynamic information geometry on the equilibrium manifold. According to the established interpretation, the negative values of the TD curvature correspond to attractive interparticle interactions. The free, noninteracting quantum theory, $R_{I}^{(Q, I I)}=0$, occurs on the level curve $h=0$, i.e.,

$$
2 \pi^{2} J\left(c_{R}-c_{L}\right)+3 S^{2}=0 .
$$

Under the current assumptions, $\left(J \geq 0, S>0, c_{R}>c_{L}\right)$, this curve is not reachable by the macro states of the black hole. Therefore, in Quevedo's approach, we always have an interacting theory.

\section{B. HPEM metric}

As an attempt to avoid extra singular points in the Quevedo thermodynamic curvature, which do not coincide with phase transitions of any type, in [41] the authors proposed an alternative information metric with a different conformal factor,

$$
d s_{\mathrm{HPEM}}^{2}=S \frac{\partial_{S} M}{\left(\partial_{J}^{2} M\right)^{3}}\left(-\partial_{S}^{2} M d S^{2}+\partial_{J}^{2} M d J^{2}\right) .
$$

Its explicit components are given by

$$
\begin{aligned}
\hat{g}^{(\mathrm{HPEM})} & =\left(\begin{array}{cc}
g_{S S}^{(\mathrm{HPEM})} & 0 \\
0 & g_{J J}^{(\mathrm{HPEM})}
\end{array}\right) \\
& =\left(\begin{array}{cc}
\frac{18 h^{5 / 2} J S\left(\sqrt{3} \mathrm{c}_{R} S-\sqrt{h}\right)}{\pi^{9} \mathrm{c}_{L}^{2}\left(\mathrm{c}_{L}-c_{R}\right)^{4} \mathrm{c}_{R}^{4}} & 0 \\
0 & \frac{3 h^{5 / 2} S\left(\sqrt{h}-\sqrt{3} \mathrm{c}_{R} S\right)}{\pi^{7} \mathrm{c}_{L}^{2}\left(\mathrm{c}_{L}-\mathrm{c}_{R}\right)^{3} \mathrm{c}_{R}^{4}}
\end{array}\right) .
\end{aligned}
$$

In this case, the denominator of the HPEM curvature is given by

$\operatorname{denom}\left(R_{I}^{(\mathrm{HPEM})}\right)=18 J^{2} S^{3}\left(\sqrt{h}-\sqrt{3} c_{R} S\right)^{3} h^{7 / 2}$.

It covers all relevant divergences of the heat capacity (3.1) including the curve (3.2), which we excluded. At first, it seems that a new spinodal curve $h=0$ is present in (5.11), but, as mentioned already, under the assumptions $(J \geq 0$, $S>0, c_{R}>c_{L}$ ), the macro states cannot lie on this curve. Therefore, the HPEM curvature is healthy from additional relevant singularities.

Contrary to Quevedo's case, here one can find the parameter region, where the Sylvester criterion holds together with $C>0$. It coincides with the region (3.4) for TD stability in $(S, J)$ space. Furthermore, in this region, $R_{I}^{\mathrm{HPEM}}$ is positive, which defines an elliptic thermodynamic information geometry on the equilibrium manifold. The latter corresponds to repulsive interparticle interactions.

\section{INFORMATION METRICS AND CONJUGATE THERMODYNAMIC POTENTIALS}

\section{A. MM metric I}

Another geometric approach to the problem of equilibrium state space of black holes was proposed by Mansoori and Mirza (MM) in [31]. The authors define a conjugate thermodynamic potential as an appropriate Legendre 
transformation of the thermal parameters in order to match the divergences of the specific heat. For example, one can consider the following conjugate potential:

$$
\begin{aligned}
K(S, \Omega) & =M(S, \Omega)-\Omega J(S, \Omega) \\
& =\frac{c_{R} \pi^{2}+3 c_{L} S \Omega(3 S \Omega-2 \pi)}{6 c_{L} \pi^{2} \Omega\left(c_{R}-c_{L}\right)},
\end{aligned}
$$

where

$$
\begin{aligned}
M(S, \Omega) & =\frac{c_{R} \pi-3 c_{L} S \Omega}{3 c_{L} \pi \Omega\left(c_{R}-c_{L}\right)}, \\
J(S, \Omega) & =\frac{c_{R} \pi^{2}-9 c_{L} S^{2} \Omega^{2}}{6 c_{L} \pi^{2} \Omega^{2}\left(c_{R}-c_{L}\right)} .
\end{aligned}
$$

In this case, the components of the information metric read

$$
\begin{aligned}
& g_{S S}^{(\mathrm{MM})}=\frac{1}{T} \frac{\partial^{2} K}{\partial S^{2}}=\frac{3 \Omega}{3 S \Omega-\pi}, \\
& g_{S \Omega}^{(\mathrm{MM})}=\frac{1}{T} \frac{\partial^{2} K}{\partial \Omega \partial S}=\frac{3 S}{3 S \Omega-\pi},
\end{aligned}
$$

$$
g_{\Omega \Omega}^{(\mathrm{MM})}=\frac{1}{T} \frac{\partial^{2} K}{\partial \Omega^{2}}=\frac{c_{R} \pi^{2}}{3 c_{L} \Omega^{3}(3 S \Omega-\pi)},
$$

where the temperature in $(S, \Omega)$ space is written by

$$
T(S, \Omega)=\frac{3 S \Omega-\pi}{\left(c_{R}-c_{L}\right) \pi^{2}} .
$$

Under the set of conditions $\{S>0, \Omega>0\}$, together with the conditions for the central charges from Sec. III, the temperature is positive if

$$
\Omega S>\frac{\pi}{3},
$$

whereas the angular momentum is non-negative if

$$
\Omega S \leq \frac{\pi}{3} \sqrt{\frac{c_{R}}{c_{L}}} .
$$

On the other hand, $C>0$ forbids the equal sign; thus we arrive at the condition for the local TD stability (3.15). The MM thermodynamic curvature yields

$$
R_{I}^{(\mathrm{MM}, I)}=\frac{3 \pi \Omega\left(c_{L} c_{R} \pi\left(9 \pi S \Omega-27 S^{2} \Omega^{2}-2 \pi^{2}\right)-c_{R}^{2} \pi^{3}-27 c_{L}^{2} S^{3} \Omega^{3}\right)}{(3 S \Omega-\pi)\left(c_{R} \pi^{2}-9 c_{L} S^{2} \Omega^{2}\right)^{2}}
$$

It exactly matches the spinodal curves (3.13) and (3.14) of the heat capacity. Thus the proposed metric is a viable choice for the description of the equilibrium space of the WAdS black hole.

The compact expression for $R_{I}^{(\mathrm{MM}, I)}$ allows us to find the conditions for its sign. For example, the MM curvature is positive if

$$
\frac{c_{L}}{c_{R}}>\frac{\pi\left(2 \pi^{2}-9 \pi S \Omega+27 S^{2} \Omega^{2}+(\pi-3 S \Omega) \sqrt{4 \pi^{2}-12 \pi S \Omega+81 S^{2} \Omega^{2}}\right)}{54 S^{3} \Omega^{3}} .
$$

On the other hand, $R_{I}^{(\mathrm{MM}, I)}$ can become negative, if

$$
\frac{c_{L}}{c_{R}}<\frac{\pi\left(2 \pi^{2}-9 \pi S \Omega+27 S^{2} \Omega^{2}+(\pi-3 S \Omega) \sqrt{4 \pi^{2}-12 \pi S \Omega+81 S^{2} \Omega^{2}}\right)}{54 S^{3} \Omega^{3}}
$$

or

$$
\frac{c_{L}}{c_{R}}>\frac{\pi\left(2 \pi^{2}-9 \pi S \Omega+27 S^{2} \Omega^{2}-(\pi-3 S \Omega) \sqrt{4 \pi^{2}-12 \pi S \Omega+81 S^{2} \Omega^{2}}\right)}{54 S^{3} \Omega^{3}} .
$$


The MM curvature is zero if expressions (6.11) and (6.12) are treated as equations separately. In any case, the values of the right-hand side of both expressions should respect Eq. (2.12).

Finally, we also find that Sylvester's criterion is compatible with the condition (3.15) for local TD stability.

\section{B. MM metric II}

Another proposal for an information metric by MM involves the Helmholtz potential in $(J, T)$ space (4.13). The new information metric is given by

$g_{T T}^{(\mathrm{MM})}=\frac{1}{T} \frac{\partial^{2} F}{\partial T^{2}}=-\frac{c_{R} \pi^{2} \sqrt{2 c_{L} J}}{\sqrt{3} T\left(1-c_{L} \pi T\left(2+\left(c_{R}-c_{L}\right) \pi T\right)\right)^{3 / 2}}$,

$$
\begin{aligned}
g_{T J}^{(\mathrm{MM})} & =\frac{1}{T} \frac{\partial^{2} F}{\partial T \partial J} \\
& =-\frac{c_{L} \pi\left(1+\left(c_{R}-c_{L}\right) \pi T\right)}{\sqrt{6} T \sqrt{c_{L} J\left(1-c_{L} \pi T\left(2+\left(c_{R}-c_{L}\right) \pi T\right)\right)}},
\end{aligned}
$$

$$
g_{J J}^{(\mathrm{MM})}=\frac{1}{T} \frac{\partial^{2} F}{\partial J^{2}}=-\frac{\sqrt{c_{L} J\left(1-c_{L} \pi T\left(2+\left(c_{R}-c_{L}\right) \pi T\right)\right)}}{2 \sqrt{6} c_{L} J^{2} T} .
$$

Now, the MM thermodynamic curvature yields

$$
R_{I}^{(\mathrm{MM}, I I)}=\frac{\sqrt{3}\left(c_{L} \pi T\left(3-\left(c_{R}-c_{L}\right)\left(c_{L} \pi T-3\right) \pi T\right)-1\right)}{\pi^{2} T \sqrt{2 c_{L} J}\left(c_{L}-c_{R}\right)\left(1-c_{L} \pi T\left(2+\left(c_{R}-c_{L}\right) \pi T\right)\right)^{3 / 2}} .
$$

It respects all relevant spinodals of the heat capacity (3.9) in $(J, T)$ space. Moreover, one notes that both MM proposals have no redundant singularities in the corresponding TD curvatures. Here, we also note that the Sylvester criterion is incompatible with the condition for local thermodynamic stability.

The sign of the second MM curvature is as follows. First, one has $R_{I}^{(\mathrm{MM}, I I)}>0$ if

$$
c_{L} \pi T\left(3-\left(c_{R}-c_{L}\right)\left(c_{L} \pi T-3\right) \pi T\right)<1 .
$$

Consequently, one has $R_{I}^{(\mathrm{MM}, I I)}<0$ in several regions. The first one is given by

$3 c_{L} \pi T \leq 1, \quad c_{L} \pi T\left(3-\left(c_{R}-c_{L}\right)\left(c_{L} \pi T-3\right) \pi T\right)>1$.

The second one is

$$
3 c_{R} \pi T<1
$$

And finally, the third region is

$$
\frac{1}{3 \pi c_{L}}<T<\frac{1}{2 \pi c_{L}}
$$

A flat information geometry, $R_{I}^{(\mathrm{MM}, I I)}=0$, occurs on the following level curve:

$$
c_{L} \pi T\left(3-\left(c_{R}-c_{L}\right)\left(c_{L} \pi T-3\right) \pi T\right)=1 .
$$

In any case, all inequalities for the sign of the curvature should respect the condition for global/local TD stability, $T<T_{c}$.

\section{COMPLEXITY GROWTH OF THE WAdS S $_{3}$ BLACK HOLE}

1In this section we compute the complexity growth rate of the $\mathrm{WAdS}_{3}$ black hole solution (2.1) within the complexity $=$ action conjecture. ${ }^{1}$ Consequently, we use the generalizations to the Lloyd bound of the rate of complexity, proposed by various authors in $[43,44]$, to find further nontrivial restrictions on the equilibrium state space parameters of the system.

The complexity $=$ volume and the complexity $=$ action conjectures for the $\mathrm{WAdS}_{3}$ solution in Einstein's theory was analyzed in [45,46], respectively. Similar studies for

\footnotetext{
${ }^{1}$ The complexity growth rate of the warped $\mathrm{AdS}_{3}$ black hole was first obtained in [42] using the first-order formalism. Unfortunately, we suspect that the result (2.28) in [42] is incorrect due to an error in the computation of the Einstein-Hilbert part of the on-shell action. The correct formula for this term should be [see Eq. (2.25) in [42]]

$$
\epsilon_{A B C} R^{A B} \wedge e^{C}=-3 \ell d t \wedge d r \wedge d \theta,
$$

which is most obvious if one compares this result directly to the on-shell part $\sqrt{-g} R=-3 \ell$. For consistency of notations we use $\ell=L$, and we have reintroduced the $\wedge$ product. One also has $\epsilon^{\operatorname{tr} \theta}=+1$, and the curvature 2-form $R^{A B}$ defined by
}

$$
R^{A B}=d \omega^{A B}+\omega^{A}{ }_{C} \wedge \omega^{C B} .
$$


the BTZ black hole were conducted in $[42,43,47,48]$. Further examples, regarding different aspects on the subject, can also be found in [49-60].

In order to compute the complexity growth rate of the $\mathrm{WAdS}_{3}$ solution from Eq. (2.1) we have to vary the TMG action (1.1) in the WDW patch:

$$
\delta I=I[t+\delta t]_{\mathrm{WDW}}-I[t]_{\mathrm{WDW}} .
$$

First, we take the variation of the Einstein-Hilbert term:

$$
\begin{aligned}
\delta I_{\mathrm{EH}} & =\frac{1}{16 \pi} \int_{0}^{2 \pi} \int_{r_{-}}^{r_{+}} \int_{t}^{t+\delta t} \sqrt{-g}\left(R+\frac{2}{L^{2}}\right) d t d r d \theta \\
& =-\frac{L\left(r_{+}-r_{-}\right)}{4} \delta t .
\end{aligned}
$$

Next, we vary the CS term:

$$
\begin{aligned}
\delta I_{C S} & =\frac{L}{96 \pi \nu} \int_{0}^{2 \pi} \int_{r_{-}}^{r_{+}} \int_{t}^{t+\delta t} 3 \nu\left(\nu^{2}-1\right) d t d r d \theta \\
& =\frac{L\left(\nu^{2}-1\right)\left(r_{+}-r_{-}\right)}{16} \delta t .
\end{aligned}
$$

To calculate the contribution coming from the boundary term $B$ one has to choose a tetrad coframe $e^{A}=e_{\mu}{ }^{A} d x^{\mu}$ of the metric $g_{\mu \nu}=e_{\mu}{ }^{A} e_{\nu}{ }^{B} \eta_{A B}, \eta=\operatorname{diag}(-1,1,1)$, namely [61]

$$
e^{0}=\frac{L}{2 \sqrt{D(r)}} d \theta=e_{\theta}{ }^{0} d \theta, \quad e^{1}=L \sqrt{D(r)} d r=e_{r}{ }^{1} d r,
$$$$
e^{2}=L d t+L M(r) d \theta=e_{t}^{2} d t+e_{\theta}^{2} d \theta .
$$

It is easy to verify that $g_{\mu \nu}=e_{\mu}{ }^{A} e_{\nu}{ }^{B} \eta_{A B}$ holds. The spin connections, $\omega^{A B}=\omega_{\mu}^{A B} d x^{\mu}$, can be calculated explicitly from the tetrad coframe by

$$
\begin{aligned}
\omega_{\mu}{ }^{A B}= & \frac{1}{2} e^{\nu A}\left(\partial_{\mu} e_{\nu}{ }^{B}-\partial_{\nu} e_{\mu}{ }^{B}\right)-\frac{1}{2} e^{\nu B}\left(\partial_{\mu} e_{\nu}{ }^{A}-\partial_{\nu} e_{\mu}{ }^{A}\right) \\
& -\frac{1}{2} e^{\rho A} e^{\sigma B}\left(\partial_{\rho} e_{\sigma C}-\partial_{\sigma} e_{\rho C}\right) e_{\mu}{ }^{C}
\end{aligned}
$$

where $e^{\nu A}=g^{\nu \lambda} e_{\lambda}{ }^{A}$ and $e_{\sigma C}=e_{\sigma}{ }^{F} \eta_{F C}$. We find

$$
\begin{aligned}
& \omega_{t}{ }^{01}=-\omega_{t}{ }^{10}=-M^{\prime}, \quad \omega_{r}{ }^{02}=-\omega_{r}{ }^{20}=-\sqrt{D} M^{\prime}, \\
& \omega_{\theta}{ }^{01}=-\omega_{\theta}{ }^{10}=M M^{\prime}-N^{\prime}, \quad \omega_{\theta}{ }^{12}=-\omega_{\theta}{ }^{21}=-\frac{M^{\prime}}{2 \sqrt{D}} .
\end{aligned}
$$

Therefore, the boundary 2-form computes to

$$
B=-\frac{L D^{\prime}}{64 \pi D^{2}} d t \wedge d \theta=\frac{L\left(\nu^{2}+3\right)\left(2 r-r_{-}-r_{+}\right)}{64 \pi} d t \wedge d \theta .
$$

Considering the above setup, the contribution from the boundary term yields

$$
\begin{aligned}
\delta I_{B}= & \frac{1}{32} \int_{t}^{t+\delta t} L\left(\nu^{2}+3\right)\left(\left.\left(2 r-r_{-}-r_{+}\right)\right|_{r_{+}}\right. \\
& \left.-\left.\left(2 r-r_{-}-r_{+}\right)\right|_{r_{-}}\right) d t=\frac{L\left(\nu^{2}+3\right)\left(r_{+}-r_{-}\right)}{16} \delta t .
\end{aligned}
$$

Finally, the result for the rate of complexity growth is

$$
\dot{C}=\frac{L}{8}\left(\nu^{2}-1\right)\left(r_{+}-r_{-}\right)=\left(2 c_{L}-c_{R}\right) \sqrt{\frac{c_{L}\left(2 J-3 c_{L} M^{2}\right)}{c_{R}\left(5 c_{L}-4 c_{R}\right)}} .
$$

Imposing $\dot{C}>0$, one recovers the condition (2.25) for local thermodynamic stability. In order to impose the Lloyd bound one also has to consider contributions from the rotation of the black hole, i.e., [43,44]

$$
\dot{C} \leq(M-\Omega J)_{+}-(M-\Omega J)_{-},
$$

where the plus and minus signs indicate calculations on the outer and the inner horizons. The latter inequality leads to a lower bound on the mass of the black hole

$$
M \geq \frac{\left(2 c_{L}-c_{R}\right) \sqrt{c_{L}}}{3 \sqrt{12 c_{R}-15 c_{L}}},
$$

which is true under condition (2.25) for local thermodynamic stability. In terms of the original gravitational parameters this bound becomes

$$
M \geq \frac{L}{3}\left(1-\frac{4}{3+\nu^{2}}\right) .
$$

For $\nu \rightarrow 1$, the mass is zero, while for large $\nu \rightarrow \infty$, one has $M \geq L / 3$.

\section{CONCLUSION}

Investigating the properties of various black hole solutions plays an important role in revealing hidden features of the elusive theory of quantum gravity. In this context, motivated by the remarkable duality between gravitational and gauge field theories, we study the thermodynamic properties of a three-dimensional warped AdS black hole solution and its dual conformal field theory. Our findings uncover the candidates for proper Riemannian metrics on the space of equilibrium states of the $\mathrm{WAdS}_{3}$ solution, together with several criteria for thermodynamic stability of the system. This allows us to constrain the possible values of the central charges from the dual field theory and the gravitational parameters from the bulk theory, thus making the duality more explicit. Our investigations were conducted mostly within the framework of thermodynamic information 
geometry, which utilizes the power of differential geometry to study statistical features of a given system.

In general, the formalism of thermodynamic information geometry treats the space of equilibrium states as a Riemaninan manifold, equipped with a proper metric and an affine connection. Here, geodesic paths correspond to quasistatic processes, while the distance between different macro states can be used to measure the probability for fluctuating between states. The parameter regions, where the geometric description breaks down, are the critical points of the system or its spinodal curves, where the corresponding thermodynamic information curvature becomes singular. In this case, from the thermodynamic point of view, near a critical point the underlying interparticle interactions become strongly correlated and the equilibrium thermodynamic considerations are no longer applicable. There are several ways to construct thermodynamic information metrics. Below we summarize our results for the warped $\mathrm{AdS}_{3}$ black hole.

In the Hessian formulation we analyzed the Ruppeiner and the Weinhold thermodynamic metrics and showed that they are inadequate for the description of the WAdS 3 black hole equilibrium state space. This is due to the occurring mismatch between the singularities of the heat capacity and the spinodal curves of the corresponding thermodynamic curvatures. Because both metrics are conformally related, with the temperature being the conformal factor, we have decided to construct another information metric based on the Hessian of the Helmholtz and Gibbs free energies. The results also showed that Hessian thermodynamic metrics are not well suited for our case. Furthermore, utilizing the properties of Gibbs and Helmholtz thermodynamic potentials, we have identified the regions of global TD stability in canonical and grand canonical ensembles, respectively, and showed that they both coincide with the local thermodynamic stability of the WAdS solution. The critical temperature (3.10), which separates thermodynamic stability from the regions of TD instability of the system, was also calculated.

On the other hand, in the case of Legendre invariant metrics, all considered thermodynamic metrics successfully manage to incorporate the relevant Davies-type critical points. Consequently they can be considered as viable metrics on the equilibrium state space of the $\mathrm{WAdS}_{3}$ black hole.

The third approach, based on conjugate thermodynamic potentials, also revealed complete agreement with the Davies critical points of the heat capacity. Here, the compact form of the thermodynamic curvatures allowed us to fully determine the regions of flat, hyperbolic, or elliptic information geometries on the space of the equilibrium states of the warped $\mathrm{AdS}_{3}$ system.

All approaches were considered together with the conditions for local and global thermodynamic stability. The latter imposed several constraints on the central charges from the dual field theory, e.g., Eqs. (2.25) and (3.4).
Several relations, namely Eqs. (2.26), (3.5), and (6.7), between the bulk gravitational parameters were also found. Additionally, in several cases, we were able to strengthen the local and global TD stability of the black hole by imposing Sylvester's criterion for positive definite metrics.

One can go even further and calculate the logarithmic correction to the entropy of the black hole, due to small thermal fluctuations around its equilibrium configuration. In Ref. [62], it was found that for any thermodynamic system, satisfying the first law, one can write the corrected form of the entropy as

$$
S^{\prime}=S+\alpha \log \left(C T^{2}\right)+\cdots
$$

Here, $S$ and $C$ are the uncorrected entropy and heat capacity, while the constant $\alpha$ depends on the underlying micro states, and dots denote higher order corrections. For example, if we choose to work in $(J, T)$ space, the corrected heat capacity of the $\mathrm{WAdS}_{3}$ black hole becomes ${ }^{2}$

$$
C^{\prime}(J, T)=T \frac{\partial S^{\prime}(J, T)}{\partial T}=C+\alpha\left(2+\frac{T}{C} \frac{\partial C}{\partial T}\right) .
$$

Local TD stability $\left(C^{\prime}>0\right)$ now requires $T<T_{c}$ and

$J>\frac{27\left(c_{L} \pi T-1\right)^{2}\left(1-c_{L} \pi T\left(2+\left(c_{R}-c_{L}\right) \pi T\right)\right) \alpha^{2}}{2 c_{L} c_{R}^{2} \pi^{4} T^{2}}$,

which reduces to $J>0$ at $\alpha=0$. On the other hand, global thermodynamic stability, imposed by the concavity $\partial^{2} F^{\prime}$ / $\partial T^{2}<0$, leads to $T<T_{c}$, together with

$$
J>\frac{27\left(1-c_{L} \pi T\left(2+\left(c_{R}-c_{L}\right) \pi T\right)\right) \alpha^{2}}{2 c_{L} c_{R}^{2} \pi^{4} T^{2}} .
$$

The latter bound on the momentum $J$ is stronger than Eq. (8.3). Hence, one concludes that the log-corrected thermodynamics of the WAdS solution respects the condition $T<T_{c}$ for global TD stability from the uncorrected case, while imposing an additional nonzero lower bound on the angular momentum $J$.

Finally, we have calculated the complexity growth of the WAdS $_{3}$ black hole within the complexity equals action proposal. The natural requirement that the complexity growth be a positive quantity turned out to be compatible with the condition (2.25) for local TD stability. Meanwhile, imposing Lloyd's bound on the complexity growth, we have found a lower bound (7.11) on the mass of the black hole.

\footnotetext{
${ }^{2}$ Note that for black holes very close to extremality $(T \rightarrow 0)$ or criticality $\left(T \rightarrow T_{c}\right.$ ) the fluctuation analysis ceases to be valid due to large quantum fluctuations.
} 


\section{ACKNOWLEDGMENTS}

The authors Seyed Ali Hosseini Mansoori and Behrouz Mirza for the valuable comments on the manuscript. This work was partially supported by Bulgarian NSF Grants No. DM18/1, No. DN18/1, and No. N28/5. T. V. gratefully acknowledges the support by the Bulgarian national program "Young Scientists and Postdoctoral Research Fellows 2019."

[1] D. Anninos, W. Li, M. Padi, W. Song, and A. Strominger, Warped AdS(3) black holes, J. High Energy Phys. 03 (2009) 130.

[2] O. Miskovic and R. Olea, Background-independent charges in topologically massive gravity, J. High Energy Phys. 12 (2009) 046.

[3] W. Li, W. Song, and A. Strominger, Chiral gravity in three dimensions, J. High Energy Phys. 04 (2008) 082.

[4] M. Gürses, Perfect fluid sources in $2+1$ dimensions, Classical Quantum Gravity 11, 2585 (1994).

[5] M. Rooman and P. Spindel, Godel metric as a squashed anti-de Sitter geometry, Classical Quantum Gravity 15, 3241 (1998).

[6] M. J. Duff, H. Lu, and C. N. Pope, $A d s_{3} \times S^{3}$ (un)twisted and squashed, and an $\mathrm{O}(2,2, \mathrm{Z})$ multiplet of dyonic strings, Nucl. Phys. B544, 145 (1999).

[7] D. Israel, C. Kounnas, and M. P. Petropoulos, Superstrings on NS5 backgrounds, deformed $\operatorname{AdS}(3)$ and holography, J. High Energy Phys. 10 (2003) 028.

[8] T. Andrade, M. Banados, R. Benguria, and A. Gomberoff, The $2+1$ Charged Black Hole in Topologically Massive Electrodynamics, Phys. Rev. Lett. 95, 021102 (2005).

[9] I. Bengtsson and P. Sandin, Anti de Sitter space, squashed and stretched, Classical Quantum Gravity 23, 971 (2006).

[10] M. Banados, G. Barnich, G. Compere, and A. Gomberoff, Three dimensional origin of Godel spacetimes and black holes, Phys. Rev. D 73, 044006 (2006).

[11] D. T. Son, Toward an AdS/cold atoms correspondence: A geometric realization of the Schrodinger symmetry, Phys. Rev. D 78, 046003 (2008).

[12] K. Balasubramanian and J. McGreevy, Gravity Duals for Non-Relativistic CFTs, Phys. Rev. Lett. 101, 061601 (2008).

[13] A. R. Brown, D. A. Roberts, L. Susskind, B. Swingle, and Y. Zhao, Complexity, action, and black holes, Phys. Rev. D 93, 086006 (2016).

[14] A. R. Brown, D. A. Roberts, L. Susskind, B. Swingle, and Y. Zhao, Holographic Complexity Equals Bulk Action?, Phys. Rev. Lett. 116, 191301 (2016).

[15] L. Susskind, Computational complexity and black hole horizons, Fortschr. Phys. 64, 44 (2016); 64, 24 (2016).

[16] L. Susskind, Entanglement is not enough, Fortschr. Phys. 64, 49 (2016).

[17] D. Stanford and L. Susskind, Complexity and shock wave geometries, Phys. Rev. D 90, 126007 (2014).

[18] S. Lloyd, Ultimate physical limits to computation, Nature (London) 406, 1047 (2000).

[19] S. Ryu and T. Takayanagi, Holographic Derivation of Entanglement Entropy from AdS/CFT, Phys. Rev. Lett. 96, 181602 (2006).
[20] H. Casini, M. Huerta, and R. C. Myers, Towards a derivation of holographic entanglement entropy, J. High Energy Phys. 05 (2011) 036.

[21] A. Lewkowycz and J. Maldacena, Generalized gravitational entropy, J. High Energy Phys. 08 (2013) 090.

[22] H. Dimov, S. Mladenov, R. C. Rashkov, and T. Vetsov, Entanglement of higher-derivative oscillators in holographic systems, Nucl. Phys. B918, 317 (2017).

[23] H. Dimov, S. Mladenov, R. C. Rashkov, and T. Vetsov, Entanglement entropy and Fisher information metric for closed bosonic strings in homogeneous plane wave background, Phys. Rev. D 96, 126004 (2017).

[24] J. E. Aman, I. Bengtsson, and N. Pidokrajt, Geometry of black hole thermodynamics, Gen. Relativ. Gravit. 35, 1733 (2003).

[25] J.-y. Shen, R.-G. Cai, B. Wang, and R.-K. Su, Thermodynamic geometry and critical behavior of black holes, Int. J. Mod. Phys. A 22, 11 (2007).

[26] R.-G. Cai and J.-H. Cho, Thermodynamic curvature of the BTZ black hole, Phys. Rev. D 60, 067502 (1999).

[27] J. E. Åman and N. Pidokrajt, Ruppeiner geometry of black hole thermodynamics, EAS Publ. Ser. 30, 269 (2008).

[28] T. Sarkar, G. Sengupta, and B. Nath Tiwari, On the thermodynamic geometry of BTZ black holes, J. High Energy Phys. 11 (2006) 015.

[29] S. A. H. Mansoori, B. Mirza, and E. Sharifian, Extrinsic and intrinsic curvatures in thermodynamic geometry, Phys. Lett. B 759, 298 (2016).

[30] S. A. H. Mansoori, B. Mirza, and M. Fazel, Hessian matrix, specific heats, Nambu brackets, and thermodynamic geometry, J. High Energy Phys. 04 (2015) 115.

[31] S. A. H. Mansoori and B. Mirza, Correspondence of phase transition points and singularities of thermodynamic geometry of black holes, Eur. Phys. J. C 74, 2681 (2014).

[32] G. Ruppeiner, Thermodynamic black holes, Entropy 20, 460 (2018).

[33] T. Vetsov, Information geometry on the space of equilibrium states of black holes in higher derivative theories, Eur. Phys. J. C 79, 71 (2019).

[34] F. Weinhold, Metric geometry of equilibrium thermodynamics, J. Chem. Phys. 63, 2479 (1975).

[35] G. Ruppeiner, Riemannian geometry in thermodynamic fluctuation theory, Rev. Mod. Phys. 67, 605 (1995).

[36] H. Quevedo, M. N. Quevedo, and A. Sanchez, Homogeneity and thermodynamic identities in geometrothermodynamics, Eur. Phys. J. C 77, 158 (2017).

[37] G. Ruppeiner, Thermodynamic curvature measures interactions, Am. J. Phys. 78, 1170 (2010). 
[38] H. Janyszek and R. Mrugala, Riemannian geometry and the thermodynamics of model magnetic systems, Phys. Rev. A 39, 6515 (1989).

[39] D. Birmingham and S. Mokhtari, Thermodynamic stability of warped $\mathrm{AdS}_{3}$ black holes, Phys. Lett. B 697, 80 (2011).

[40] V. A. Lubarda, On the gibbs conditions of stable equilibrium, convexity and the second-order variations of thermodynamic potentials in nonlinear thermoelasticity, Int. J. Solids Struct. 45, 48 (2008).

[41] S. H. Hendi, S. Panahiyan, B. Eslam Panah, and M. Momennia, A new approach toward geometrical concept of black hole thermodynamics, Eur. Phys. J. C 75, 507 (2015).

[42] M. Ghodrati, Complexity growth in massive gravity theories, the effects of chirality, and more, Phys. Rev. D 96, 106020 (2017).

[43] R.-G. Cai, S.-M. Ruan, S.-J. Wang, R.-Q. Yang, and R.-H. Peng, Action growth for AdS black holes, J. High Energy Phys. 09 (2016) 161.

[44] H. Huang, X.-H. Feng, and H. Lu, Holographic complexity and two identities of action growth, Phys. Lett. B 769, 357 (2017).

[45] R. Auzzi, S. Baiguera, and G. Nardelli, Volume and complexity for warped AdS black holes, J. High Energy Phys. 06 (2018) 063.

[46] R. Auzzi, S. Baiguera, M. Grassi, G. Nardelli, and N. Zenoni, Complexity and action for warped AdS black holes, J. High Energy Phys. 09 (2018) 013.

[47] S. A. Hosseini Mansoori and M. M. Qaemmaqami, Complexity growth, butterfly velocity and black hole thermodynamics, arXiv:1711.09749.

[48] M. M. Qaemmaqami, Complexity growth in minimal massive 3D gravity, Phys. Rev. D 97, 026006 (2018).

[49] A. Bhattacharya and S. Roy, Holographic entanglement entropy, subregion complexity and fisher information metric of 'black' non-SUSY D3 brane, arXiv:1807.06361.

[50] D. Carmi, S. Chapman, H. Marrochio, R. C. Myers, and S. Sugishita, On the time dependence of holographic complexity, J. High Energy Phys. 11 (2017) 188.
[51] K. Nagasaki, Complexity of $\mathrm{AdS}_{5}$ black holes with a rotating string, Phys. Rev. D 96, 126018 (2017).

[52] M. Reza Tanhayi, R. Vazirian, and S. Khoeini-Moghaddam, Complexity growth following multiple shocks, Phys. Lett. B 790, 49 (2019).

[53] S. A. Hosseini Mansoori, V. Jahnke, M. M. Qaemmaqami, and Y.D. Olivas, Holographic complexity of anisotropic black branes, arXiv:1808.00067.

[54] P. A. Cano, R. A. Hennigar, and H. Marrochio, Complexity Growth Rate in Lovelock Gravity, Phys. Rev. Lett. 121, 121602 (2018).

[55] S. Chapman, H. Marrochio, and R. C. Myers, Holographic complexity in Vaidya spacetimes. Part II, J. High Energy Phys. 06 (2018) 114.

[56] S. Chapman, H. Marrochio, and R. C. Myers, Holographic complexity in Vaidya spacetimes. Part I, J. High Energy Phys. 06 (2018) 046.

[57] D. Carmi, R. C. Myers, and P. Rath, Comments on holographic complexity, J. High Energy Phys. 03 (2017) 118.

[58] N. S. Mazhari, D. Momeni, S. Bahamonde, M. Faizal, and R. Myrzakulov, Holographic complexity and fidelity susceptibility as holographic information dual to different volumes in AdS, Phys. Lett. B 766, 94 (2017).

[59] D. Momeni, S. A. H. Mansoori, and R. Myrzakulov, Holographic complexity in gauge/string superconductors, Phys. Lett. B 756, 354 (2016).

[60] D. Momeni, M. Faizal, S. Alsaleh, L. Alasfar, A. Myrzakul, and R. Myrzakulov, Thermodynamic and holographic information dual to volume, Eur. Phys. J. C 78, 765 (2018).

[61] B. Chen and Z.-b. Xu, Quasi-normal modes of warped black holes and warped AdS/CFT correspondence, J. High Energy Phys. 11 (2009) 091.

[62] S. Das, P. Majumdar, and R. K. Bhaduri, General logarithmic corrections to black hole entropy, Classical Quantum Gravity 19, 2355 (2002). 